\title{
Variation of genomic DNA methylation in the nitrate reductase gene of sibling tobacco (Nicotiana tabacum) cultivars
}

\author{
S.L. Fu, Z.X. Tang, L. Liu, L.M. Lu and Y.B. Huang \\ Agronomic College, Sichuan Agricultural University, Chengdu, China \\ Corresponding author: Y.B. Huang \\ E-mail: huangyubi123@sohu.com
}

Genet. Mol. Res. 11 (2): 1169-1177 (2012)

Received August 1, 2011

Accepted December 20, 2011

Published May 7, 2012

DOI http://dx.doi.org/10.4238/2012.May.7.2

\begin{abstract}
To better understand genomic DNA methylation in sibling plant cultivars, methylation-sensitive amplification polymorphism analysis was used to investigate two sibling tobacco cultivars, Yunyan85 and Yunyan87, and their two parents, K326 and Yunyan No. 2. Differences in the degree of genomic DNA methylation were found among the four tobacco cultivars. Compared with parents, the two sibling cultivars had fewer methylated sites. Twenty-nine methylationsensitive amplification polymorphism fragments that exhibited methylation alteration in the four tobacco cultivars were recovered and sequenced. BLAST (nucleotide BLAST) searches showed that two of the 29 sequences have $99 \%$ similarity with nucleotides 1442-1694 of the nia-1 gene and the other 27 sequences contain GC, CAAT or TATA box. The nitrate reductase genes from Yunyan87, K326 and Yunyan No. 2 were found to be identical; however, the third intron of the nitrate reductase gene from Yunyan85 was different compared to the third introns of Yunyan87, K326 and Yunyan No. 2. We conclude that methylation alteration of promoter regions could be responsible for the different phenotypes in tobacco and that introns of the nitrate reductase
\end{abstract}


gene can vary as a result of intra-species crossing in tobacco.

Key words: Tobacco; Sibling cultivars; Methylation; NR gene; Intron variation

\section{INTRODUCTION}

It is well known that methylation is involved in epigenetic processes (Weinhold, 2006). In the higher plant genome, about $20-50 \%$ of cytosines are methylated, among which about $90 \%$ methylated sites lie in ' $\mathrm{CpG}$ ' dinucleotides or ' $\mathrm{CpNpG}$ ' trinucleotides (Madlung et al., 2002; Chan et al., 2005; Xiao et al., 2006). DNA methylation plays an important role in gene expression (Assaad et al., 1993; Matzke and Matzke, 1998; Akimoto et al., 2007). The cytosine methylation of tobacco (Nicotiana tabacum) 18S, 5.8S and 26S rRNA genes in leaf calli and in regenerated plants and their progeny have been studied (Koukalova et al., 2005). The DNA methylation patterns in transgenic tobacco plants have been extensively investigated (Fojtova et al., 2003; Oh et al., 2009). These previous studies have given us direct evidence that genomic DNA methylation has a close relationship with gene expression. During plant breeding processes, some sibling cultivars have been developed. These sibling cultivars are the best models for research on the relationship between DNA methylation and gene expression. Characterizing the methylation alteration of sibling plant cultivars will provide a better understanding of the gene expression phenotype, because they often have a similar genetic background and genomic structure (Zhang et al., 2008). Yang et al. (2011) have reported the different genomic DNA methylation pattern between two sibling tobacco cultivars with similar genomic structure. The GC methylation has already been discovered in the nitrate reductase (NR) gene.

NR is an $\mathrm{NAD}(\mathrm{P}) \mathrm{H}$-dependent enzyme and it is responsible for the conversion of nitrate to nitrite, which is considered to be the rate-limiting step in nitrogen assimilation. NR has been shown to be the subject of multifactorial control at the level of both gene expression and posttranslational modification (Kaiser and Huber, 1994; Kaiser et al., 1999). Analysis of NR genes in plants showed that their expressions were primarily regulated at the transcriptional level (Campbell, 1999). Some genes for nitrate reductase have been cloned from fungi (Fu and Marzluf, 1987; Okamoto et al., 1991), algae (Falcão et al., 2010) and higher plants (Choi et al., 1989; Vaucheret et al., 1989; Wilkinson and Crawford, 1991; Wu et al., 1995) and their evolutionary relationships were investigated (Zhou and Kleinhofs, 1996). The NR genes from fungi, algae and higher plants show substantial sequence similarity, particularly within functional domains, and large variations in GC content at the third codon position and in intron number (Zhou and Kleinhofs, 1996). The intron positions are different between fungi and plants, but conserved within these groups (Zhou and Kleinhofs, 1996). Although NR genetics, biochemistry and molecular biology have been intensively investigated, little is known about the NR gene variation between sibling tobacco cultivars.

In the present study, two sibling tobacco cultivars and their parents were first used to investigate the methylation pattern at the 'CCGG/GGCC' site. The sequences involved in methylation contained the NR gene sequence. Thereafter, the NR genes were cloned from the two sibling tobacco cultivars and their parents, and the variation in the NR gene between the two sibling tobacco cultivars was determined; the result are discussed. 


\section{MATERIAL AND METHODS}

\section{Plant materials}

Two sibling tobacco cultivars, Yunyan85 and Yunyan87, and their parents, tobacco cultivar K326 and Yunyan No. 2, were used. The pedigree of the two sibling tobacco cultivars was described by Tan et al. (1997) and Li et al. (2001). The two sibling cultivars have been proven to have highly similar genomic structure (Yang et al., 2011).

\section{DNA extraction}

Tobacco cultivars Yunyan85, Yunyan87, K326 and Yunyan No. 2 were planted in Yibin, Sichuan, China, and their leaves were collected on July 13, 2010. Genomic DNA was isolated from leaf tissue according to Tang et al. (2008).

\section{Methylation-sensitive amplification polymorphism (MSAP) analysis}

$H p a \mathrm{II} / M s p \mathrm{I}$ and $E c o$ RI restriction enzymes were used for MSAP analysis. The MSAP procedures were performed according to Zhang et al. (2008). The EcoRI and HpaII/MspI adaptors, the pre-selective primers, and the selective primer combinations are listed in Table 1. In MSAP procedures, repeats were carried out and patterns resulting from two independent digestions were compared for each sample. In addition, for MSAP gels, the upper part and the lower part of the gel, where resolution was not satisfactory, were not used for band scoring. Only stable and repeatable patterns were retained for analysis.

Table 1. Sequences of methylation-sensitive amplification polymorphism (MSAP) adaptors and pre-selective and selective primers used in this study.

\section{Adaptors}

EcoRI-adaptors-F EcoRI-adaptors-R

HpaII/MspI-adaptors-F

HpaII/MspI-adaptors-R

Pre-selective primers

EcoRI-A

HpaII/MspI-T

Selective primer combination

EcoRI-AAG $+H p a \mathrm{II} / M s p \mathrm{I}-\mathrm{TCAG}$

$E c o$ RI-AAG $+H p a \mathrm{II} / M s p \mathrm{I}-\mathbf{T C T G}$

EcoRI-AAC $+H p a \mathrm{II} / M s p \mathrm{I}-\mathrm{TCTT}$

EcoRI-AAC $+H p a \mathrm{II} / M s p \mathrm{I}-\mathrm{TCAT}$

\author{
5'-CTCGTAGACTGCGTACC-3' \\ 5'-AATTGGTACGCAGTC-3' \\ 5'-GATCATGAGTCCTGCT-3' \\ 5'-CGAGCAGGACTCATGA-3' \\ 5'-GACTGCGTACCAATTCA-3' \\ 5'-ATCATGAGTCCTGCTCGGT-3 \\ $E c o$ RI-AAC $+H p a I I / M s p I-T C T G$ \\ EcoRI-AAG $+H p a \mathrm{II} / M s p \mathrm{I}-\mathrm{TCAA}$ \\ EcoRI-ACA $+H p a \mathrm{II} / M s p \mathrm{I}-\mathrm{TCAA}$
}

The bold letters indicate the selective nucleotides.

\section{Isolation and sequencing of polymorphic methylated fragment}

The polymorphic MSAP fragments were isolated from polyacrylamide gels, re-amplified by polymerase chain reaction (PCR), and sequenced. The procedures were performed according to Zhang et al. (2008). 


\section{Isolation of the NR gene}

Four sets of primer pairs, Nia1-1 (5'-GCCACTCCTCCGAAAATAAAT-3'; 5'-CTGTCTGATTCTTGGGTGGTG-3'), Nia1-2 (5'-GTTTCCAGTACGAATGATAAT-3'; 5'-GTGATCTAGTGTGCAAACTTG-3'), Nia1-3 (5'-CAAGTTTGCACACTAGATCAC-3'; 5'-ATCTTGACAACCAACTCGAAG-3'), and Nia1-4 (5'-TTGGTGAACTCCTAACTACTG3'; 5'-AAGACACATGAGCACAATGTT-3'), were designed according to the sequence of nitrate reductase gene nia-1 (GenBank accession No. X14058), which was cloned from $N$. tabacum. The genomic DNA of the four tobacco cultivars was used as template for PCR amplification. The LA Taq polymerase (TaKaRa) was used in the PCR amplification. The PCR cycling included $94^{\circ} \mathrm{C}$ for $5 \mathrm{~min}$, followed by 35 cycles of $94^{\circ} \mathrm{C}$ for $40 \mathrm{~s}, 60^{\circ} \mathrm{C}$ for $1 \mathrm{~min}$ and $72^{\circ} \mathrm{C}$ for $2 \mathrm{~min}$, and then a final extension step at $72^{\circ} \mathrm{C}$ for $10 \mathrm{~min}$. PCR products were separated on $1 \%$ agarose gels. The target fragments were recovered, cloned and sequenced according to Tang et al. (2008). For each target fragment, at least three clones were randomly selected out for sequencing. The sequences cloned from each tobacco cultivar were then ligated into a complete gene sequence. Sequences were analyzed using the DNAMAN software (version 5.2.2; Lynnon Biosoft) and Clustal $\mathrm{X}$ (Version 1.81). In addition, the complete gene sequences were analyzed using GENSCAN Web Server (http://genes.mit.edu/GENSCAN.html).

\section{RESULTS}

\section{Genomic DNA methylation extent in sibling cultivars and their parents}

Because HpaII will not cut if either the outer or the inner cytosine of the 'CCGG/ GGCC' site is fully (double-strand) methylated, whereas, MspI will not cut if the external cytosine is fully or hemi- (single-strand) methylated, the methylation states of the cytosine at 'CCGG/GGCC' sites would lead to a differential cleavage by the two isoschizomers. Thus, the band pattern from PCR amplification can reflect the methylation status at a certain site. The seven pairs of $E c o \mathrm{RI}+H p a \mathrm{II} / M s p \mathrm{I}$ selective primer combinations produced legible and reproducible fragments at 162 sites. Of these 162 sites, 82 (50.62\%), 66 (40.74\%), 72 (44.44\%), and $50(30.86 \%)$ methylated sites were detected in Yunyan No. 2, K326, Yunyan85, and Yunyan87, respectively (Table 2). This result indicates that apparent differences in the extent of genomic DNA methylation existed among the tobacco cultivars used in this study. Further analysis showed that there were differences between full-methylation and hemi-methylation extent in all four tobacco cultivars (Table 2). The number of full-methylated sites were 55 (33.95\%), 25 $(15.43 \%), 35(21.60 \%)$, and $10(6.17 \%)$ in Yunyan No. 2, K326, Yunyan85, and Yunyan87, respectively, and the corresponding hemi-methylated sites were 27 (16.67\%), 41 (25.31\%), $37(22.84 \%)$, and $40(24.69 \%)$. In both Yunyan No. 2 and Yunyan87, significant differences existed between full-methylation and hemi-methylation. Compared to Yunyan No. 2, the fullmethylated sites of Yunyan85 and Yunyan87 were decreased, whereas the hemi-methylated sites of the two cultivars were increased. Compared to K326, the full-methylated sites were increased in Yunyan85 and were greatly decreased in Yunyan87; however, the hemi-methylated sites were decreased in both sibling cultivars. Between Yunyan85 and Yunyan87, the fullmethylated sites were significantly different. This result indicates that the extent of genomic DNA methylation could exhibit great differences between sibling tobacco cultivars. 
Table 2. Number of bands amplified using eight methylation-sensitive amplification polymorphism (MSAP) selective primer combinations in the tobacco cultivars.

\begin{tabular}{|c|c|c|c|c|c|c|c|c|c|}
\hline \multirow[t]{3}{*}{ Primer combination } & \multirow[t]{3}{*}{ Amplification site } & \multirow{2}{*}{\multicolumn{2}{|c|}{$\begin{array}{c}\text { Yunyan No. } 2 \\
\text { Methylated site }\end{array}$}} & \multicolumn{2}{|c|}{ K326 } & \multirow{2}{*}{\multicolumn{2}{|c|}{$\begin{array}{c}\text { Yunyan85 } \\
\text { Methylated site }\end{array}$}} & \multirow{2}{*}{\multicolumn{2}{|c|}{$\begin{array}{c}\text { Yunyan87 } \\
\text { Methylated site }\end{array}$}} \\
\hline & & & & \multicolumn{2}{|c|}{ Methylated site } & & & & \\
\hline & & Full & Hemi & Full & Hemi & Full & Hemi & Full & Hemi \\
\hline $\mathrm{E}-\mathrm{AAC}+\mathrm{H} / \mathrm{M}-\mathrm{TCTG}$ & 22 & 10 & 0 & 1 & 7 & 3 & 5 & 1 & 8 \\
\hline $\mathrm{E}-\mathrm{AAC}+\mathrm{H} / \mathrm{M}-\mathrm{TCTT}$ & 26 & 7 & 6 & 8 & 7 & 2 & 4 & 0 & 7 \\
\hline E-AAC+H/M-TCAT & 25 & 7 & 6 & 1 & 5 & 6 & 5 & 3 & 1 \\
\hline E-AAG+H/M-TCAA & 26 & 8 & 6 & 6 & 6 & 6 & 7 & 1 & 11 \\
\hline $\mathrm{E}-\mathrm{ACA}+\mathrm{H} / \mathrm{M}-\mathrm{TCAA}$ & 27 & 6 & 6 & 4 & 3 & 7 & 7 & 1 & 5 \\
\hline E-AAG+H/M-TCTG & 18 & 11 & 1 & 2 & 4 & 7 & 3 & 3 & 6 \\
\hline $\mathrm{E}-\mathrm{AAG}+\mathrm{H} / \mathrm{M}-\mathrm{TCAG}$ & 18 & 6 & 2 & 3 & 9 & 4 & 6 & 1 & 2 \\
\hline \multirow[t]{2}{*}{ Total } & 162 & 55 & 27 & 25 & 41 & 35 & 37 & 10 & 40 \\
\hline & & 82 & & 66 & & 72 & & 50 & \\
\hline \multirow[t]{2}{*}{ Rate of methylated site (\%) } & & $33.95 \%$ & $16.67 \%$ & $15.43 \%$ & $25.31 \%$ & $21.60 \%$ & $22.84 \%$ & $6.17 \%$ & $24.69 \%$ \\
\hline & & $50.62 \%$ & & $40.74 \%$ & & $44.44 \%$ & & $30.86 \%$ & \\
\hline
\end{tabular}

\section{Sequencing of polymorphic MSAP fragments}

Twenty-nine MSAP fragments that exhibited methylation alteration in Yunyan85, Yunyan87, K326, and Yunyan No. 2 were recovered and sequenced. The BLAST (nucleotide BLAST) searches showed that two of the 29 sequences had $99 \%$ similarity with the nucleotides 1442-1694 bp of the nia-1 gene (GenBank accession No. X14058). In addition, the two sequences were respectively cloned from K326 and Yunyan87, and they showed 100\% similarity. All remaining 27 sequences were found to contain a GC, CAAT or TATA box.

\section{Characterization of the NR gene in the four tobacco cultivars}

From each of the four tobacco cultivars, three NR gene sequences were cloned and sequenced. A total of 12 NR gene sequences were obtained. Sequence alignment analysis indicated that the nine NR gene sequences cloned from Yunyan No. 2, K326 and Yuanyan87 have $99.8-100 \%$ similarity with each other, and the three NR gene sequences cloned from Yunyan 85 have $100 \%$ similarity with each other. However, the NR gene sequence of Yunyan 85 exhibited differences from that of the other three cultivars. Therefore, only the NR gene sequences of K326 and Yunyan85 were deposited into GenBank, and the NR gene sequence of K326 could represent the ones of Yunyan No. 2 and Yunyan87. The NR gene sequences of K326 and Yunyan85 were designated as K326-nia-1 (GenBank accession No. JN384019) and Yunyan85-nia-1 (GenBank accession No. JN384020), respectively.

The lengths of K326-nia-1 and Yunyan85-nia-1 are 5441 and 5306 bp, respectively, and they have $96.9 \%$ similarity. Both of the two sequences have four exons and three introns (Table 3). It can be noted that in both K326-nia-1 and Yunyan85-nia-1 sequences, the promoter region is $235-300 \mathrm{bp}$ and the transcription initiation codon is 445-447 bp; however, 5133-5135 $\mathrm{bp}$ is the stop codon in the K326-nia-1 sequence, while 4998-5000 bp in the Yunyan85-nia-1 sequence is the stop codon. The difference between the two sequences was mainly caused by the variation in the third intron of Yunyan85-nia-1 (Table 3). The third intron of Yunyan85nia-1 (3019-3671 bp) is shorter than that of K326-nia-1 (3019-3806 bp) because of nucleotide elimination (Figure 1). Between the two sequences, the main nucleotide difference also oc- 
curred in the third intron (Figure 1). Besides the nucleotide changes in the third intron, A-G or $\mathrm{C}-\mathrm{T}$ transitions have also occurred at another 16 nucleotide positions. However, the deduced peptide sequences of the two genes have $100 \%$ similarity.

Table 3. Structure of the nitrate reductase gene cloned from K326 and Yunyan85.

\begin{tabular}{|c|c|c|c|c|c|c|c|}
\hline Sequence & $\begin{array}{l}\text { Length } \\
\text { (bp) }\end{array}$ & $\begin{array}{l}\text { Promoter } \\
\text { region (bp) }\end{array}$ & $\begin{array}{l}\text { Transcription initiation } \\
\text { codon (bp) }\end{array}$ & Exons (bp) & Introns (bp) & $\begin{array}{l}\text { Poly(A)-signal } \\
\text { (bp) }\end{array}$ & $\begin{array}{l}\text { Stop codon } \\
\quad \text { (bp) }\end{array}$ \\
\hline K326-nia-1 & 5441 & $235-330$ & $445-447$ & $\begin{array}{r}445-1456 \\
1732-1872 \\
2786-3018 \\
3806-5134\end{array}$ & $\begin{array}{l}1457-1731 \\
1873-2785 \\
3019-3806\end{array}$ & 5163-5168 & $5133-5135$ \\
\hline Yunyan85-nia-1 & 5306 & $235-330$ & $445-447$ & $\begin{array}{r}445-1456 \\
1732-1872 \\
2786-3018 \\
3672-5000 \\
\end{array}$ & $\begin{array}{l}1457-1731 \\
1873-2785 \\
3019-3671\end{array}$ & $5028-5033$ & $4998-5000$ \\
\hline
\end{tabular}

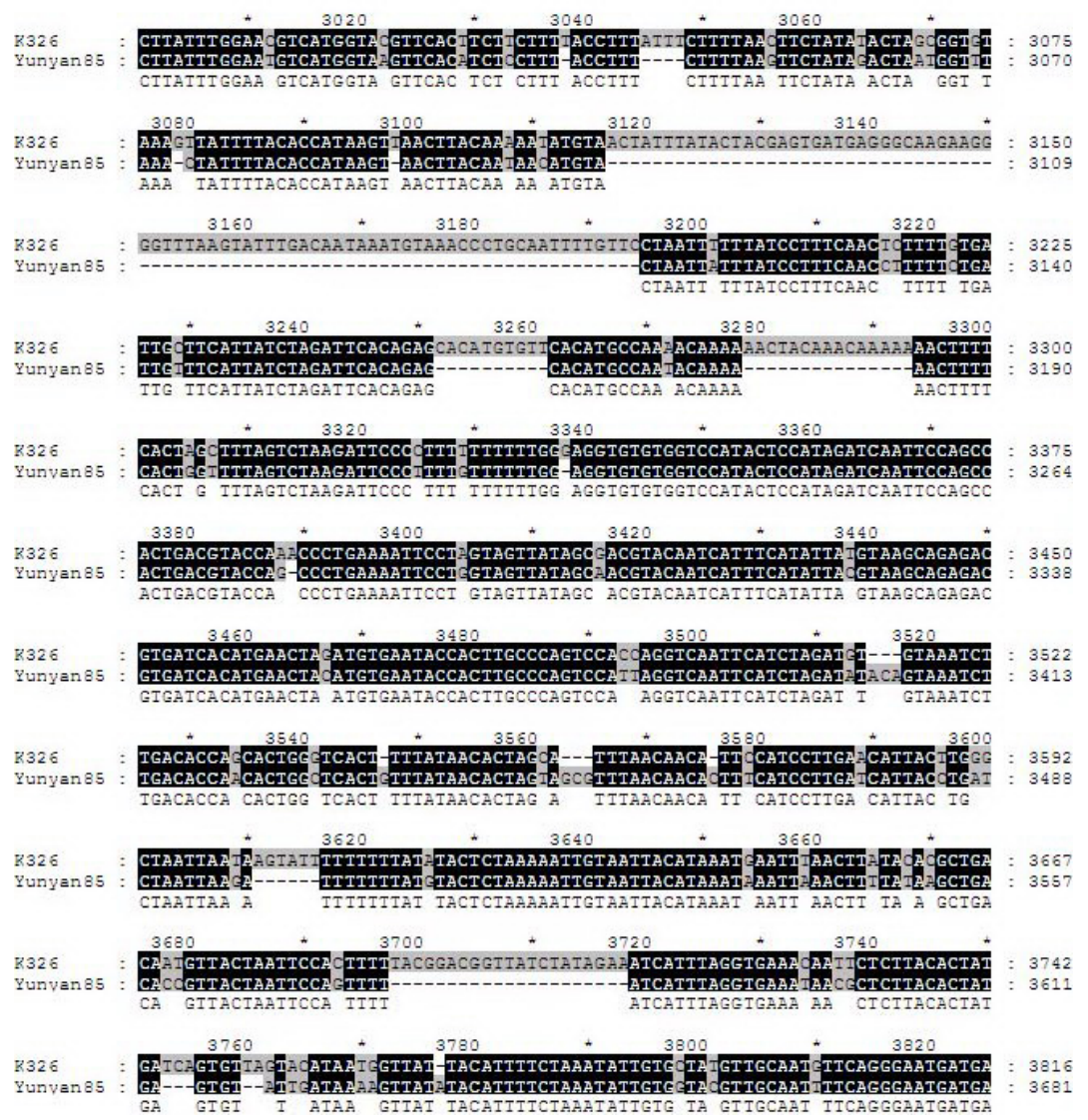

Figure 1. Alignment of the two NR genes of K326 and Yunyan85 using Clustal X. Only 3001-3816/3681 bp is shown because the differences of the two genes occurred mainly in this region. K326 and Yunyan 85 indicate K326nia-1 and Yunyan85-nia-1, respectively. Note that the third intron of the NR gene of Yunyan85 is shorter than that of K326 because of nucleotide elimination. 


\section{DISCUSSION}

\section{Genomic DNA methylation in sibling tobacco cultivars}

The samples with identical parents are the best models for research on the relationship between DNA methylation and gene expression. Particular DNA methylation could lead to the discordance of monozygotic (MZ) twins (Singh et al., 2002). Epigenetics has been associated with MZ twin discordance for common diseases (Poulsen et al., 1999; Bjornsson et al., 2004). Alterations in methylation patterns in sibling wheat cultivars have been discovered and these alterations may be responsible for the different phenotypes among sibling wheat cultivars (Zhang et al., 2008). In these sibling wheat cultivars, the alterations in methylation patterns mainly affected repetitive DNA sequences (Zhang et al., 2008). Many previous studies have also indicated that the main target sequences for methylation mutation are repetitive DNA sequences in hybrid plants (Shaked et al., 2001; Madlung et al., 2002, 2005). Yang et al. (2011) reported that the sibling tobacco cultivars Yunyan85 and Yunyan 87 have similar primary genomic structure; however, there was a difference in methylation extent of genomic DNA between the two cultivars, and the sequences involved in the methylation alteration mainly included promoter regions. In the present study, Yunyan85 and Yunyan 87 were planted at different times and in different places from that described by Yang et al. (2011). However, the results obtained in this study are very similar with the ones gotten by Yang et al. (2011), that is, the genomic DNA methylation extent exhibited great differences between the two sibling tobacco cultivars and almost all the sequences involved in methylation alteration contain a TATA, CAAT or GC box. It has been proposed that the biological function of DNA methylation is involved in gene silencing, often being associated with hypermethylation of promoter sequences (Paszkowski and Whitham, 2001; Bird, 2002). Both the results of the present study and those obtained by Yang et al. (2011) indicate that methylation alteration of promoter regions may be mainly responsible for the gene expression phenotype of tobacco, and this may not be affected by environment.

\section{Variation in the NR gene in sibling tobacco cultivars}

It has already been reported that the three introns in NR genes from Oryza sativa (rice), Phaseolus vulgaris (bean), Lycopersicon esculentum (tomato), and N. tabacum (tobacco) are located precisely at the same position (Zhou and Kleinhofs, 1996). In the present study, the intron from Yunyan 85 and that from the three other cultivars are also located at the same position. That is, all four NR genes of the four tobacco cultivars used in this study have three introns and each intron started from the same position (Table 3). However, because of nucleotide elimination, the third intron of the NR gene from Yunyan85 is shorter than that from Yunyan87, K326 and Yunyan No. 2 (Figure 1). This result indicates that the intron of the NR gene in tobacco will change in crosses between the two tobacco cultivars. It is known that introns have a relationship with gene evolution (Fedorova and Fedorov, 2003). Information on the dynamics of intron evolution has come mainly from studies within species or comparisons of different species. Previous reports have, for the most part, examined the historical mutation events of introns (Liaud et al., 1992; Zhou and Kleinhofs, 1996). The variation of the third intron of the NR gene of Yunyan85 indicates that intron evolution could occur immediately after a cross between intra-plant species. 


\title{
ACKNOWLEDGMENTS
}

\author{
Research supported by the Sichuan Tobacco Monopoly Bureau(Contract\#200703009).
}

\section{REFERENCES}

Akimoto K, Katakami H, Kim HJ, Ogawa E, et al. (2007). Epigenetic inheritance in rice plants. Ann. Bot. 100: 205-217. Assaad FF, Tucker KL and Signer ER (1993). Epigenetic repeat-induced gene silencing (RIGS) in Arabidopsis. Plant Mol. Biol. 22: 1067-1085.

Bird A (2002). DNA methylation patterns and epigenetic memory. Genes Dev. 16: 6-21.

Bjornsson HT, Fallin MD and Feinberg AP (2004). An integrated epigenetic and genetic approach to common human disease. Trends Genet. 20: 350-358.

Campbell WH (1999). Nitrate reductase structure, function and regulation: bridging the gap between biochemistry and physiology. Annu. Rev. Plant Physiol. Plant Mol. Biol. 50: 277-303.

Chan SWL, Henderson IR and Jacobsen SE (2005). Gardening the genome: DNA methylation in Arabidopsis thaliana. Nat. Rev. Genet. 6: 351-360.

Choi HK, Kleinhofs A and An GH (1989). Nucleotide sequence of rice nitrate reductase genes. Plant Mol. Biol. 13: 731733.

Falcão RV, Oliveira MC and Colepicolo P (2010). Molecular characterization of nitrate reductase gene and its expression in the marine red alga Gracilaria tenuistipitata (Rhodophyta). J. Appl. Phycol. 22: 613-622.

Fedorova L and Fedorov A (2003). Introns in gene evolution. Genetica 118: 123-131.

Fojtova M, Houdt HV, Depicker A and Kovarik A (2003). Epigenetic switch from posttranscriptional to transcriptional silencing is correlated with promoter hypermethylation. Plant Physiol. 133: 1240-1250.

Fu YH and Marzluf GA (1987). Molecular cloning and analysis of the regulation of Nit-3, the structural gene for nitrate reductase in Neurospora crassa. Proc. Natl. Acad. Sci. U. S. A. 84: 8243-8247.

Kaiser WM and Huber SC (1994). Post-translational regulation of nitrate reductase in higher plants. Plant Physiol. 106: 817-821.

Kaiser WM, Weiner H and Huber SC (1999). Nitrate reductase in higher plants: A case study for transduction of environmental stimuli into control of catalytic activity. Physiol. Plantarum 105: 384-389.

Koukalova B, Fojtova M, Lim KY, Fulnecek J, et al. (2005). Dedifferentiation of tobacco cells is associated with ribosomal RNA gene hypomethylation, increased transcription, and chromatin alterations. Plant Physiol. 139: 275-286.

Li YP, Wang YK, Ma WG and Tan CL (2001). Breeding and selecting of a new flue-cured tobacco variety Yunyan87 and its characteristics. Chin. Tob. Sci. 4: 42-43 (In Chinese with English abstract).

Liaud MF, Brinkmann H and Cerff R (1992). The $\beta$-tubulin gene family of pea: primary structures, genomic organization and intron-dependent evolution of genes. Plant Mol. Biol. 18: 639-651.

Madlung A, Masuelli RW, Watson B, Reynolds SH, et al. (2002). Remodeling of DNA methylation and phenotypic and transcriptional changes in synthetic Arabidopsis allotetraploids. Plant Physiol. 129: 733-746.

Madlung A, Tyagi AP, Watson B, Jiang HM, et al. (2005). Genomic changes in synthetic Arabidopsis polyploids. Plant J. 41: 221-230.

Matzke MA and Matzke AJM (1998). Epigenetic silencing of plant transgenes as a consequence of diverse cellular defence responses. Cell Mol. Life Sci. 54: 94-103.

Oh YJ, Chung H, Yu JG and Park YD (2009). Newly developed MSAP analysis reveals the different polymorphism patterns in transgenic tobacco plants with the dsRNA MET1 gene. Plant Biotechnol. Rep. 3: 139-145.

Okamoto PM, Fu YH and Marzluf GA (1991). Nit-3, the structural gene of nitrate reductase in Neurospora crassa: nucleotide sequence and regulation of mRNA synthesis and turnover. Mol. Gen. Genet. 227: 213-223.

Paszkowski J and Whitham SA (2001). Gene silencing and DNA methylation processes. Curr. Opin. Plant Biol. 4: 123 129.

Poulsen P, Kyvik KO, Vaag A and Beck-Nielsen H (1999). Heritability of type II (non-insulin-dependent) diabetes mellitus and abnormal glucose tolerance - a population-based twin study. Diabetologia 42: 139-145.

Shaked H, Kashkush K, Ozkan H, Feldman M, et al. (2001). Sequence elimination and cytosine methylation are rapid and reproducible responses of the genome to wide hybridization and allopolyploidy in wheat. Plant Cell 13: 1749-1759.

Singh SM, Murphy B and O'Reilly R (2002). Epigenetic contributors to the discordance of monozygotic twins. Clin. Genet. 62: 97-103.

Tan CL, Li YP, Wang YK, Ma WG, et al. (1997). Breeding and selecing a new variety of flue cured tobacco Yunyan 85 
and its characteristics. Chin. Tob. Sci. 1: 7-10 (In Chinese with English abstract).

Tang ZX, Fu SL, Ren ZL, Zhou JP, et al. (2008). Variations of tandem repeat, regulatory element, and promoter regions revealed by wheat-rye amphiploids. Genome 51: 399-408.

Vaucheret H, Vincentz M, Kronenberger J, Caboche M, et al. (1989). Molecular cloning and characterisation of the two homologous genes coding for nitrate reductase in tobacco. Mol. Gen. Genet. 216: 10-15.

Weinhold B (2006). Epigenetics: the science of change. Environ. Health Perspect. 114: A160-A167.

Wilkinson JQ and Crawford NM (1991). Identification of the Arabidopsis CHL3 gene as the nitrate reductase structural gene NIA2. Plant Cell 3: 461-471.

Wu SC, Lu Q, Kriz AL and Harper JE (1995). Identification of cDNA clones corresponding to two inducible nitrate reductase genes in soybean: analysis in wild-type and nr1 mutant. Plant Mol. Biol. 29: 491-506.

Xiao WY, Custard KD, Brown RC, Lemmon BE, et al. (2006). DNA methylation is critical for Arabidopsis embryogenesis and seed viability. Plant Cell 18: 805-814.

Yang CY, Huang YB, Tang ZX, Lu LM, et al. (2011). Analysis of DNA methylation variation in sibling tobacco (Nicotiana tabacum) cultivars. Afr. J. Biotechnol. 10: 874-881.

Zhang Y, Liu ZH, Liu C, Yang ZJ, et al. (2008). Analysis of DNA methylation variation in wheat genetic background after alien chromatin introduction based on methylation-sensitive amplification polymorphism. Chin. Sci. Bull. 53: 58-69.

Zhou JZ and Kleinhofs A (1996). Molecular evolution of nitrate reductase genes. J. Mol. Evol. 42: 432-442. 